\title{
DINÁMICA DE LA REGENERACIÓN DE PINUS MONTEZUMAE POSTERIOR A UN INCENDIO Y A QUEMA PRESCRITA
}

\section{PINUS MONTEZUMAE REGENERATION DYNAMICS AFTER A FOREST FIRE AND A PRESCRIBED BURN}

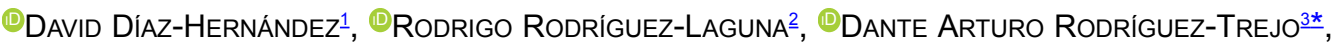

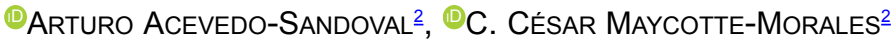

\begin{abstract}
IInstituto de Ciencias Básicas e Ingeniería, Universidad Autónoma del Estado de Hidalgo, Tulancingo, Hidalgo, México. ${ }^{2}$ Instituto de Ciencias Agropecuarias, Universidad Autónoma del Estado de Hidalgo, Tulancingo, Hidalgo, México. ${ }^{3}$ División de Ciencias Forestales, Universidad Autónoma Chapingo, Chapingo, Estado de México, México.
\end{abstract}

*Autor para la correspondencia: dantearturo@yahoo.com

\section{Resumen}

Antecedentes: Se han hecho observaciones empíricas de que el fuego favorece la regeneración natural en muchos pinares. Sin embargo, esto se ha demostrado en pocas especies experimentalmente.

Preguntas y/o Hipótesis: ¿Favorece el fuego la regeneración de la especie estudiada?, ¿cómo cambia la densidad de ésta los primeros años? Especies de estudio/Descripción de datos/Modelo matemático: Pinus montezumae Lamb. Densidad de la regeneración y profundidad de la capa de hojarasca. Correlación de Pearson, pruebas de Kruskal-Wallis y de Wilcoxon.

Sitio y años de estudio: Ejido Santo Tomás, Singuilucan, Hidalgo. De 2011 a 2013.

Métodos: A 9, 21 y 27 meses de una quema prescrita (QP) y a 30, 42 y 48 meses de un incendio (IF) y áreas testigo sin quemar (T), se muestrearon 56 parcelas de $1 \mathrm{~m}^{2}$ por tratamiento.

Resultados: Se obtuvo una correlación de Pearson negativa $(r=-0.648)$ significativa $(P \leq 0.01)$, la cual demostró que a mayor espesor de hojarasca, menor densidad de regeneración. En casi todas las comparaciones por año entre pares de tratamientos, en las tres evaluaciones, hubo diferencias significativas $(P<0.05)$. La densidad de regeneración en el primer muestreo fue 492,625 (IF), $402,875(\mathrm{QP})$, y 12,375 ha ${ }^{-1}$ (T). Para la tercera evaluación se hallaron 160,000 (IF), 84,643 (QP) y 0 ha $^{-1}$ (T).

Conclusiones: El fuego favoreció la regeneración de P. montezumae.

Palabras clave: Espesor de hojarasca, fuego, plántulas de pino.

\section{Abstract}

Background: Empirically, it has been observed that fire favors the regeneration in many pine forests. However, few studies have demonstrated this experimentally.

Questions and/or Hypotheses: ¿Does the fire favor the studied species regeneration? ¿how does it change the regeneration density along the first years?

Studied species/data description/Mathematical model: Pinus montezumae Lamb. Seedling density, litter thickness. Pearson correlation, Kruskal-Wallis and Wilcoxon tests.

Study site and dates: Ejido Santo Tomás, Singuilucan, Hidalgo state, Mexico. From 2011 to 2013.

Methods: Fifty-six-1 $\mathrm{m}^{2}$ plots per treatment were sampled 9, 21 and 27 months after a prescribed fire (QP) and 30, 42 and 48 months after a forest fire (IF) as well as unburned controls.

Results: There was a negative Pearson correlation $(r=-0.648, P \leq 0.01)$, showing that as it decreases the thickness of the litter, the density of regeneration increases. When comparing couples of treatments per year along the three evaluations, almost all showed significant differences $(P<0.05)$. In the first evaluation, the regeneration density was 492,625 (IF), 402,875 (QP) and 12,375 ha-1 (T). In the third evaluation was found 160,000 (IF), 84,643 (QP) and $0 \mathrm{ha}^{-1}(\mathrm{~T})$.

Conclusions: The fire favored the regeneration of $P$. montezumae.

Keywords: fire, litter thickness, pine seedlings. 
Si bien el fuego puede ser un factor de degradación, es también una herramienta ampliamente utilizada en la agricultura y la silvicultura; la investigación ecológica ha demostrado que los incendios han sido parte de la dinámica de los ecosistemas forestales y del ambiente evolutivo de su biota (Rodríguez-Trejo 1996, Jardel-Peláez et al. 2006, Bonilla-Vichot et al. 2009).

Los incendios forestales han estado afectando la vegetación terrestre desde hace más de 400 millones de años (Scott 2018). Por ello, desde entonces, y hasta el presente, hay muchos ecosistemas en los cuales dominan las especies adaptadas al fuego (Scott et al. 2014). Por su relación con el fuego, los ecosistemas se dividen en: mantenidos por el fuego (evolucionaron bajo los efectos de este, que ayuda a mantener su composición, estructura y procesos, sin él cambian a otra cosa, pues la sucesión avanza); sensibles al fuego (no evolucionaron bajo su influencia, el fuego los degrada y se tiene que reiniciar la sucesión ecológica); influenciados por el fuego (con unas especies adaptadas, y otras no, a este factor); e independientes del fuego (aquí los incendios no se pueden presentar) (Hardesty et al. 2005). En México, ejemplos de ecosistemas mantenidos por el fuego son la gran mayoría de pinares y encinares, diversos matorrales, pastizales $\mathrm{y}$ palmares y otros; entre los ecosistemas sensibles al fuego están las selvas altas y medianas, así como los bosques mesófilos de montaña; como parte de los influenciados están diversas selvas bajas y vegetación de galería; y entre los independientes al fuego, los matorrales en condiciones de aridez extrema (Rodríguez-Trejo 2008, Rodríguez-Trejo et al. 2019).

La regeneración natural se lleva a cabo en sitios donde las condiciones edafoclimáticas son adecuadas para desencadenar la germinación y que puedan establecerse las nuevas plántulas que ocuparán el lugar de los árboles al alcanzar su límite de vida (Vela-Correa et al. 2007, FloresGarnica \& Benavides-Solorio 2009); Marroquín-Flores et al. (2007) refieren que en la restauración ecológica hay dos caminos para recuperar el orden histórico de la comunidad: a través de la regeneración natural cuando tome lugar, y a través de una rápida restauración con siembra de semillas, plantación de árboles producidos en vivero o por otros medios. Por el contrario, en condiciones de alta acumulación de material en descomposición y densa vegetación del sotobosque (como gramíneas), se crean barreras que limitan el establecimiento de plántulas recién germinadas, pues su radícula no logra establecer contacto con el suelo mineral para establecerse o, al secarse la cama de hojarasca, también se deshidrata la plántula. Asimismo, se ha investigado que la hojarasca de algunos encinos puede contener algunas sustancias alelopáticas que inhiben la germinación de semillas de pino (Miller 2000). De acuerdo con lo anterior, el fuego crea camas adecuadas para la repoblación de especies como la mayoría de los pinos, que se establecen más fácilmente sobre el suelo mineral. Esto obedece a que al consumir el fuego gramíneas u hojarasca, elimina la barrera física que representan, se liberan nutrimentos como parte de las cenizas, que proveerán a las plántulas de pino y también se volatiliza a posibles productos alelopáticos presentes; asimismo, durante la época de lluvias la humedad en el suelo mineral puede ser mayor que cuando está bajo una capa más gruesa de hojarasca (Smith et al. 1997, Miller 2000). Estos últimos autores también señalan que el color oscuro de la superficie quemada absorbe más radiación infrarroja y genera condiciones más cálidas que favorecen a las plántulas durante el invierno, además de que acelera la mineralización.

De manera similar, entre las investigaciones que han demostrado que la regeneración de pinos es favorecida por el fuego, están las de Platt (1999) para P. palustris Mill. en Norteamérica; Bonilla-Vichot et al. (2009) para P. tropicalis Morelet en Pinar del Río, Cuba; De las Heras-Ibáñez et al. (2011) para $P$. halepensis Mill. en la Península Ibérica y Hancock et al. (2009) para P. sylvestris L. en Escocia. Sin embargo, en México son muy escasos este tipo de trabajos y $P$. montezumae no ha sido investigado formalmente bajo esta óptica.

Desde luego, incendios forestales intensos o quemas prescritas intensas, además de producir mortalidad en el arbolado y potencialmente poder afectar los conos con sus semillas, dejan temporalmente desprotegido el suelo que puede erosionarse en sitios con pendiente. Si el fuego es de bajas intensidad y severidad, no se afectará al arbolado y la vegetación del sotobosque se recuperará más rápidamente, reduciéndose el peligro de erosión (DeBano et al. 1998, Rodríguez-Trejo 2014), en particular en terrenos con poca pendiente o planos, como el del área de estudio.

Por lo tanto, es necesario el uso de quemas prescritas, con técnicas que maximicen los impactos positivos, como el reciclaje de la materia orgánica para favorecer la disponibilidad de nutrimentos, regeneración, reducción de peligro de incendios más intensos, hábitat adecuado para fauna, heterogeneidad para favorecer la diversidad, y los usos silvícolas, de conservación y tradicionales. Además, minimizan los impactos negativos de incendios, como mortalidad de árboles y fauna, deforestación, erosión y contaminación (Rodríguez-Trejo 1996, González-Rosales \& Rodríguez-Trejo 2004).

Se debe señalar que en los tipos de vegetación mantenidos por el fuego, las especies tienen adaptaciones al fuego. En $P$. montezumae Lamb. estas adaptaciones incluyen regeneración en áreas incendiadas, estado cespitoso, rápido crecimiento en altura luego del estado cespitoso, rebrotación en la base, recuperación de copa afectada, y corteza gruesa que protege al cambium (Rodríguez-Trejo \& Fulé 2003, Rodríguez-Trejo 2014). 
Por lo anterior, el objetivo del presente estudio es analizar el efecto del fuego en el establecimiento de la regeneración natural de Pinus montezumae, además de evaluar la dinámica de la regeneración establecida a lo largo de tres años. La hipótesis es que el fuego remueve los detritos y favorece la regeneración.

\section{Materiales y métodos}

Ubicación del área de estudio. El presente estudio se realizó en el Ejido Santo Tomás. Se ubica dentro del Municipio de Singuilucan, Hidalgo (igura 1). El predio se localiza al sur de Singuilucan, aproximadamente $1 \mathrm{~km}$ al suroeste de la localidad Francisco I. Madero, entre los paralelos $19^{\circ} 53^{\prime}$ $54.94 " \mathrm{~N}$ a $19^{\circ} 52^{\prime \prime} 00.25^{\prime}$ N $\mathrm{N}$, y entre los meridianos $98^{\circ}$ 28'08.88" O a $98^{\circ} 31^{\prime}$ '27.71" O. Presenta un clima de tipo $C$ (W1)(w) templado subhúmedo con lluvias en verano, con precipitación media anual de $752.3 \mathrm{~mm}$ y una temperatura media anual de $15.2{ }^{\circ} \mathrm{C}$ (INEGI 1992). Muestra un intervalo altitudinal de entre 2,860 y $3,200 \mathrm{~m} \mathrm{snm}$; y el terreno tiene una pendiente promedio $<12 \%$.

Descripción del área de estudio. Las evaluaciones se llevaron a cabo en tres zonas con vegetación dominante de Pinus montezumae Lamb.; una zona perturbada por un incendio forestal superficial (IF) que ocurrió en el mes de marzo del año 2009 y cubrió 150 ha del ejido; otra zona donde se aplicó una quema prescrita (QP) de bajas intensidad y severidad sobre 2 ha en diciembre de 2010; y una tercera zona sin quemar por lo menos en 27 años (Comunicación personal Alfonso Díaz Jiménez, 2012, ex Presidente del Comisariado Ejidal de Santo Tomás, Zempoala, Hgo.), utilizada como testigo (T), de la que se consideró una superficie de 12 ha. Dichos tratamientos muestran condiciones similares en pendiente del terreno, densidad de arbolado en monte alto y altura sobre el nivel del mar, pero diferentes en densidad de arbustos del sotobosque, exposición y fecha de ocurrencia (Tabla 1). Cabe señalar que, aunque se desconocía si había regeneración en la zona incendiada, por lo menos en el área estudiada o era muy escasa o fue consumida por el fuego. Respecto a las áreas testigo y de quema prescrita, no había regeneración previa, razón por la cual se aplicó este último tratamiento de quema.

Muestreo de hojarasca. Se midió el espesor de la hojarasca, con regla, como una variable que limita el establecimiento de la regeneración. Se realizó una medición por sitios de $1 \mathrm{~m}^{2}$, de los utilizados para evaluar la regeneración, como se señala en el siguiente párrafo. Esta variable solo se obtuvo durante la primera de las tres evaluaciones al renuevo.

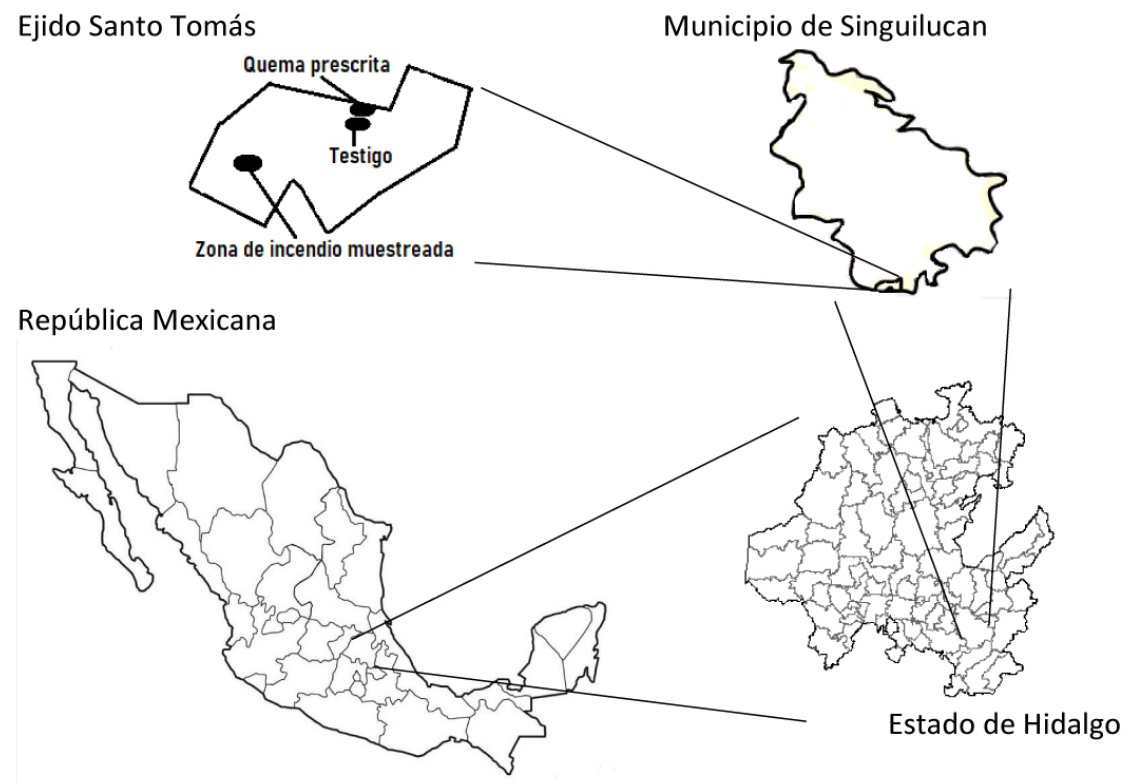

Figura 1. Localización de la zona de estudio (Ejido Santo Tomás, Singuilucan, Hidalgo). 
Tabla 1. Densidades y variables ambientales del sitio en donde se aplicaron los tratamientos.

\begin{tabular}{ccccccc}
\hline Tratamiento & $\begin{array}{c}\text { Densidad monte alto } \\
\left(\text { árboles ha } \mathbf{~ h a ~}^{-1}\right.\end{array}$ & $\begin{array}{c}\text { Densidad sotobosque } \\
\left(\text { (arbustos ha }^{-1}\right)\end{array}$ & Pendiente (\%) & Exp. Asnm (m) & $\begin{array}{c}\text { Fecha de ocurrencia o } \\
\text { realización }\end{array}$ \\
\hline Quema Prescrita (QP) & 108 & 110 & 6 & $\mathrm{~N}$ & 3,005 & Dic. 2010 (9 meses*) \\
Incendio Forestal (IF) & 99 & 27 & 9 & $\mathrm{NW}$ & 2,990 & Mar. 2009 (2.5 años*) \\
Testigo sin quemar (T) & 108 & 117 & 6 & $\mathrm{~N}$ & 3,025 & - \\
\hline
\end{tabular}

*Tiempo entre la quema prescrita o el incendio y la primera evaluación de regeneración.

Muestreo de regeneración. En los meses de enero y febrero de 2011, se dio una gran producción (año semillero) y dispersión de semilla de $P$. montezumae en la región donde se encuentra el área de estudio. Con la finalidad de tener la mayor representatividad de la regeneración natural establecida después del paso del fuego en los tratamientos de quema prescrita e incendio forestal, así como en la superficie sin quemar, se realizó un muestreo sistemático con 56 parcelas permanentes de $1 \mathrm{~m}^{2}$ (Mostacedo \& Fredericksen 2000) en cada uno de los tratamientos (168 parcelas en total), de acuerdo con la siguiente distribución. En el área incendiada y en el testigo, sobre un trayecto lineal, cada $50 \mathrm{~m}$ se ubicaron puntos a partir de los cuales, hacia los cuatro puntos cardinales, se ubicaron las parcelas de $1 \mathrm{~m}^{2}$. En el caso del área de quema prescrita, por su menor superficie, el trayecto fue en varias líneas. La evaluación se hizo en tres épocas diferentes (primera evaluación en septiembre de 2011; segunda evaluación en septiembre de 2012 y tercera evaluación en marzo de 2013), para conocer las densidades de regeneración y su dinámica poblacional a través del tiempo. Se contabilizó el número de individuos, tomando como regeneración a los brinzales y a las plántulas no lignificadas producto de la germinación de semillas liberadas después del incendio y de la aplicación de la quema prescrita. Aquellas plántulas que por sus características físicas presentaron daños relevantes $o$ condiciones decadentes, como fragilidad y decoloración, fueron excluidas debido a su escasa presencia $<3 \%$ en la población.

Radiación solar. Para conocer bajo qué condiciones de radiación solar se estableció la regeneración, durante el segundo muestreo de regeneración (septiembre de 2012), se tomaron 14 fotografías hemisféricas para cada uno de los tratamientos quema prescrita e incendio forestal (28 en total), ubicadas al azar. Las fotografías digitales se obtuvieron con una cámara digital con lente hemisférica (de "ojo de pescado"), y fueron analizadas con el programa HemiView v.2.1., para obtener cielo visible (tanto por uno), las radiaciones solares bajo dosel: difusa, directa y total $\left(\mathrm{MJ} \mathrm{m}^{-2}\right.$ año $\left.^{-1}\right)$, además del índice de área foliar $\left(\mathrm{m}^{2} \mathrm{~m}^{-2}\right)$ en los puntos de toma de cada foto.

Análisis estadísticos. A los datos por tratamiento de cada registro de regeneración, así como a los de profundidad de la capa de hojarasca, se les aplicó la prueba de normalidad con el procedimiento univariado (PROC UNIVARIATE) de SAS. Como no mostraron normalidad (se tuvo $p<0.05$ para las pruebas de Kolmogorov-Smirnov, Cramer von-Mises y Anderson-Darling), se les aplicó la prueba no paramétrica de Kruskal-Wallis y luego se comparó entre pares de tratamientos mediante la prueba no paramétrica de Wilcoxon (ambas con PROC NPAR1WAY) (López et al. 2001), siempre por periodo de evaluación.

También se compararon los diferentes indicadores de radiación e índice de área foliar obtenidos en incendio forestal y quema prescrita. Para ello se aplicó la prueba de t, considerando si había o no igualdad de varianzas (PROC TTEST de SAS). Finalmente se aplicó la prueba de correlación de Pearson a los datos de densidad de regeneración y profundidad de la capa de hojarasca, en la primera medición, mediante el procedimiento correlación (PROC CORR) de SAS. En todos los casos se empleó el programa SAS (Statistical Analysis System), v. 9 para microcomputadoras (SAS Institute 2012).

\section{Resultados}

Espesor de la hojarasca. La prueba de Wilcoxon mostró que hay diferencias en la profundidad de la cama de hojarasca entre quema prescrita o bien incendio forestal y el testigo no quemado $(P<0.0001$ en ambos casos), pero no entre los tratamientos quema prescrita $\mathrm{e}$ incendio $(P=0.3191)$. El espesor en el IF y la QP son inferiores en 81 y $78 \%$, respectivamente, comparados con el T $(100 \%)$ (Figura 2).

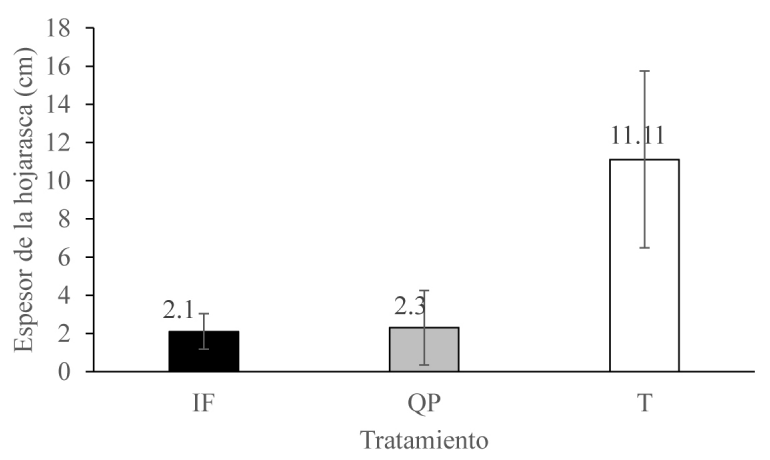

Figura 2. Espesor medio de hojarasca en quema prescrita (QP), incendio forestal (IF) y testigo (T). La barra de error indica desviación estándar. 
Con los valores promedio de las variables de regeneración y espesor de hojarasca obtenidas en la primera evaluación, se obtuvo una correlación de Pearson negativa y significativa $(r=-0.648, P \leq 0.001)$, lo cual demuestra que a medida que disminuye el espesor de la hojarasca, aumenta la densidad de regeneración natural, y viceversa.

Dinámica de la regeneración. La aplicación de la prueba de Kruskal-Wallis arrojó diferencias $(P<0,0001)$ en densidad de la regeneración entre tratamientos en la primera evaluación, de septiembre de 2011 (a 9 meses de la quema prescrita y a 30 meses del incendio). Al comparar entre quema prescrita o incendio forestal y testigo no quemado, con la prueba de Wilcoxon, se hallaron diferencias significativas $(P \leq 0.0001)$. Sin embargo, no se hallaron diferencias entre quema prescrita e incendio forestal $(P=0.0773)$ ( $\underline{\text { Figura } 3})$.

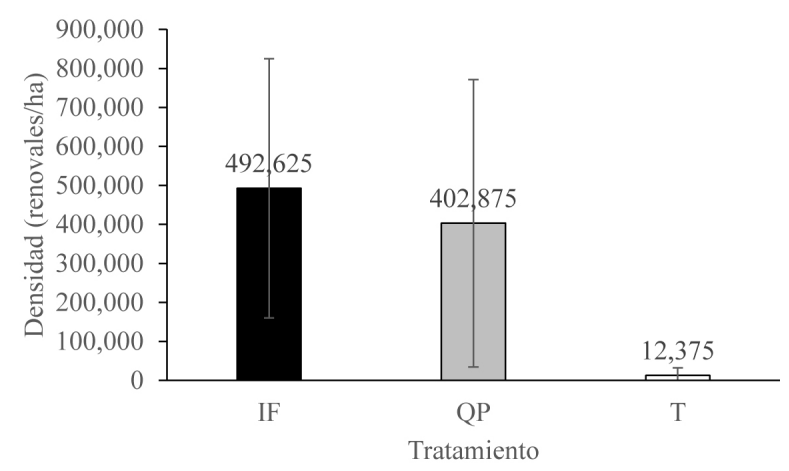

Figura 3. Densidad de regeneración de $P$. montezumae a 9 meses de la aplicación de quema prescrita (QP) y a 30 meses de un incendio forestal (IF). $\mathrm{T}=$ testigo.

Los resultados de la segunda evaluación en septiembre de 2012, mostraron diferencias estadísticamente significativas entre los tratamientos con la prueba de Kruskal-Wallis $(P<0.0001)$, así como entre todos los pares de comparaciones para los tres tratamientos (Wilcoxon, $P<0.0001$ ) (Figura 4), con una disminución de 250,485 plántulas ha $^{-1}(50.9 \%)$ en el incendio forestal; 308,946 plántulas ha $^{-1}$ en la quema prescrita $(76.7 \%)$ y 12,018 plántulas ha ${ }^{-1}(97.1 \%)$ en el área testigo, en relación con las existentes en la primera evaluación.

La tercera evaluación, a 27 meses de la quema y a 48 del incendio, realizada en marzo de 2011, mostró diferencias significativas entre los tratamientos (Kruskal-Wallis, $P<0.0001$ ) (Figura 5), exhibiendo las siguientes reducciones en la densidad, con respecto a la primera evaluación, 332,625 plántulas ha ${ }^{-1}(67.5 \%)$ en el incendio forestal; 316,000 plántulas ha $\mathrm{ha}^{-1}(79.0 \%)$ en la quema prescrita; y 12,000 plántulas ha ${ }^{-1}(100 \%)$ en la zona testigo. La comparación entre los tratamientos de quema prescrita $\mathrm{e}$ incendio forestal (Wilcoxon), arrojó una $P=0.0235$, mientras que el resto de pares de comparaciones tuvieron una $P<0.0001$. La reducción de la regeneración natural durante el periodo de estudio, se muestra por tratamiento en la Figura 6.

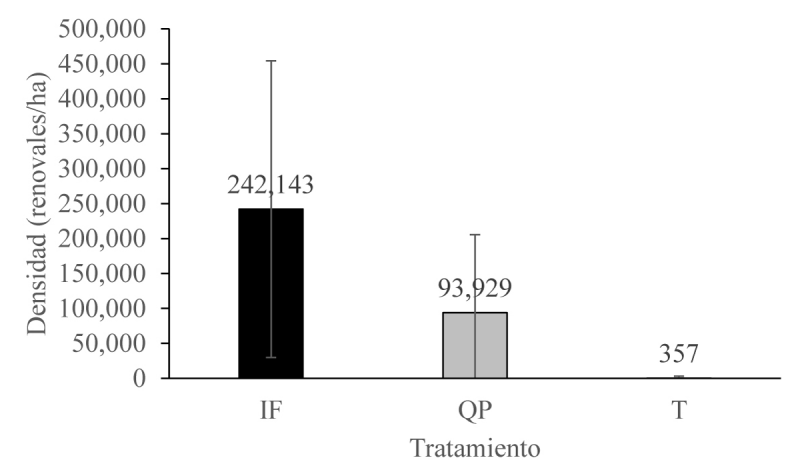

Figura 4. Densidad de regeneración de $P$. montezumae a 21 meses de la aplicación de quema prescrita (QP), y a 42 meses de un incendio forestal (IF). $\mathrm{T}=$ testigo.

Radiación solar. Se hallaron diferencias significativas en la radiación solar directa y en la radiación solar total entre quema prescrita e incendio forestal, con mayores niveles en el segundo. Los niveles de radiación resultaron adecuados para favorecer la regeneración (Tabla 2).

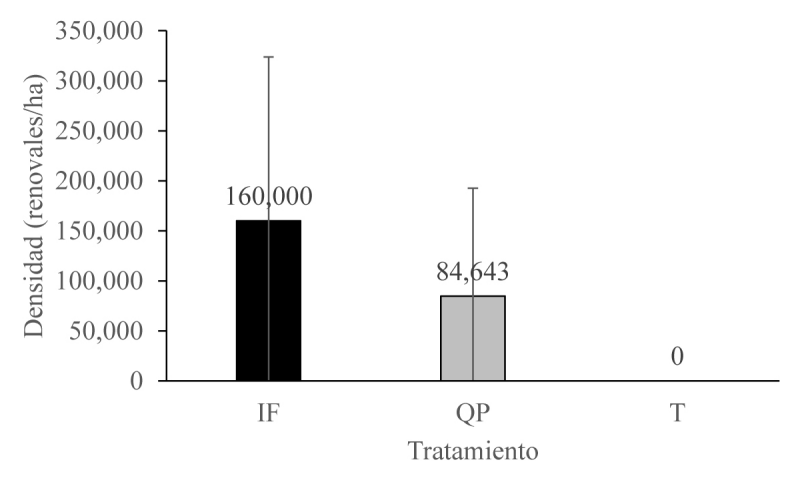

Figura 5. Densidad de regeneración de $P$. montezumae a 27 meses de la aplicación de quema prescrita (QP), y a 48 meses de un incendio forestal (IF). $\mathrm{T}=$ testigo.

\section{Discusión}

Aunque los incendios forestales están aumentando su extensión y severidad globalmente, su papel en la regeneración natural y artificial, para la recuperación del bosque, se conoce pobremente. En masas de Pinus ponderosa P.Lawson \& C.Lawson del sureste de Estados Unidos, solo la mitad de las áreas estudiadas se regeneraron bien a lo largo de 10 años después de un incendio forestal de alta severidad (Ouzts et al. 2015). 


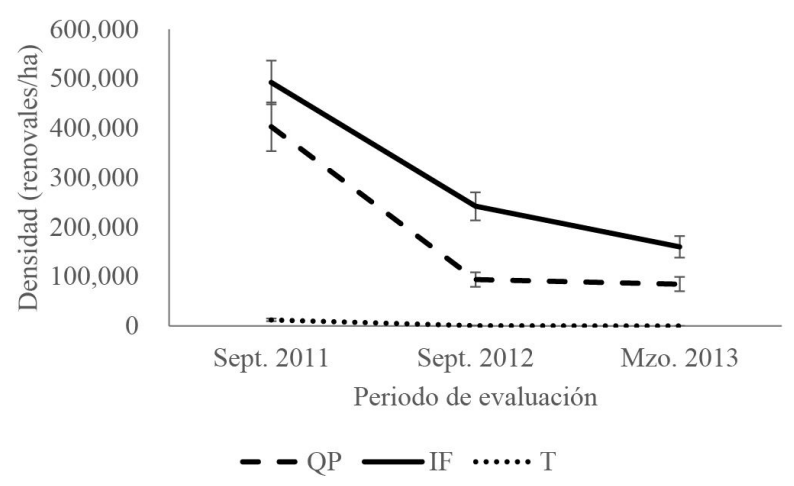

Figura 6. Dinámica de la regeneración natural de $P$. montezumae, en incendio forestal (IF), quema prescrita (QP) y testigo (T). La barra de error indica error estándar.

El fuego remueve barreras físicas como gramíneas, arbustos, hojarasca y materiales leñosos, que dificultan el contacto de la semilla de P. patula Schltdl. \& Cham. (VelaGálvez 1980), P. oocarpa var. ochoterenae Martínez (Juárez-Martínez \& Rodríguez-Trejo 2004) y de pinos norteamericanos (Miller 2000) con el suelo mineral. Ejemplo de reducción de materiales vegetales con el fuego, es el de Martínez-Becerra (2006) quien utilizando quemas prescritas experimentales logró una reducción de $66 \%$ de hojarasca, en bosque de $P$. tropicalis Morelet en Cuba. Con los resultados obtenidos en el presente estudio, se observa que efectivamente el fuego remueve las barreras que impiden que la semilla de P. montezumae llegue a suelo mineral, facilitando con esto su germinación y establecimiento, de acuerdo con Pérez et al. (2012), quienes indican que de la espesura del mantillo depende que las semillas de Pinus cubensis Griseb. puedan llegar al suelo mineral, germinar y establecerse.

Tabla 2. Pruebas de igualdad de varianzas y de $\mathrm{t}$ para los indicadores de radiación.

\begin{tabular}{|c|c|c|c|c|}
\hline Indicador & QP & IF & $\begin{array}{c}P \text { igualdad } \\
\text { varianzas }\end{array}$ & $P$ \\
\hline RSFbd (MJ mn ${ }^{-2}$ ano $\left.^{-1}\right)$ & 430.0 & 436.0 & 0.838 & 0.742 \\
\hline $\operatorname{RSDbd}\left(\mathrm{MJ} \mathrm{m}^{-2} \mathrm{año}^{-1}\right)$ & $1,468.0$ & 1881.0 & 0.664 & 0.018 \\
\hline RSTbd (MJ m² año $\left.{ }^{-1}\right)$ & $1,898.0$ & 2317.0 & 0.562 & 0.024 \\
\hline CV $(\%)$ & 29.3 & 28.7 & 0.234 & 0.648 \\
\hline $\operatorname{IAF}\left(\mathrm{m}^{2} \mathrm{~m}^{-2}\right)$ & 1.174 & 1.2 & 0.085 & 0.7312 \\
\hline
\end{tabular}

Si bien la hojarasca es una barrera para la regeneración natural, una vez que se establecen las plántulas luego de un incendio o quema prescrita, se comienza a acumular nuevamente y puede favorecer inicialmente la supervivencia de las plántulas. Bonnet et al. (2005), hallaron que $P$. ponderosa se regeneró mejor entre hojarasca parcialmente afectada por el fuego, pues ayuda a retener humedad. De forma similar, entre los factores que favorecen la regeneración de $P$. pinaster Aiton en Europa, están una cobertura herbácea y de hojarasca, que reduce la depredación y proporcionan mejores condiciones microambientales (Ruano et al. 2015).

En lo que se refiere a la densidad de la regeneración en la zona testigo, prácticamente entre el transcurso de la primera y segunda evaluación, se presentó la mayor mortandad, atribuyendo esta disminución a la competencia intraespecífica y a que la raíz de la plántula nunca tuvo contacto con el suelo mineral, ya que su germinación se dio prácticamente en la hojarasca, perdiendo así la posibilidad de prosperar en la zona mineral del piso forestal.

Por otra parte, el ambiente posterior a la quema implica condiciones de humedad y temperatura más favorables para la regeneración, además el fuego ayuda a la transformación de la materia orgánica en nutrientes asimilables y elimina temporalmente fitopatógenos que podrían afectar a las plántulas (DeBano et al. 1998). Estos últimos autores refirieren que las cenizas contienen nutrientes disponibles en la superficie del suelo (como potasio, calcio, magnesio y fósforo), con la posible excepción del nitrógeno, que favorecen el establecimiento y desarrollo inicial de la regeneración.

Se sabe que, en general, la supervivencia de la regeneración de los pinos aumenta conforme incrementa la radiación solar (hasta valores límite), como hallaron $\underline{\mathrm{Li} \& \mathrm{Li}}$ (2003) para P. koraiensis Siebold \& Zucc., o BermúdezRodríguez (2018) en P. hartwegii Lindl. del Nevado de Toluca, especie que prefiere los claros para regenerarse (si bien alguna cobertura les puede favorecer), lo que también es consistente con el presente trabajo, con mayor densidad de regeneración y radiación en el área de IF que en la de QP. La diferencia entre estas dos últimas, puede deberse a que la reducción de detritos en mayor en incendios forestales, por su mayor intensidad y severidad, que en quemas prescritas (Scott et al. 2014).

De forma semejante al presente trabajo, Juárez-Martínez \& Rodríguez-Trejo (2004) hallaron que las áreas quemadas a alta severidad por incendios forestales resultaron con mayor regeneración de $P$. oocarpa var. ochoterenae, que las áreas quemadas a baja severidad y el testigo, a 2 y 4 años del siniestro. Aunque los resultados no son idénticos en el presente estudio y el anterior citado, sí evidencian que la remoción de obstáculos para las semillas mejora la regeneración. La regeneración post fuego es común en 
bosques de pino de Estados Unidos, por ejemplo, en masas de $P$. ponderosa, donde además hay esquemas de quemas prescritas con ese y otros objetivos (Arno 2000). Sin embargo, las condiciones del micrositio con frecuencia influencian esta respuesta post-fuego. Después de un incendio de alta severidad en el suroeste de Estados Unidos, $P$. ponderosa var. scopulorum Engelm. se regeneró bien en sitios de mayor altitud y más húmedos, a menos de $150 \mathrm{~m}$ de fuentes de semilla. En cambio, en las zonas más secas y bajas, con fuentes de semilla lejanas, la regeneración resultó mucho menor o ausente (Haffey et al. 2018). De forma similar, masas de P. contorta var. latifolia Engelm. ex S.Watson afectadas por el descortezador Dendroctonus ponderosae Hopkins y por un incendio de alta severidad que cubrió 10,000 ha en Canadá, mostraron mejor regeneración conforme los sitios eran más húmedos y la severidad del fuego menor (Egger et al. 2009). Las quemas prescritas normalmente se hacen a baja severidad y así contribuyen a favorecer la regeneración de pinos, como $P$. ponderosa en Nuevo México (Zachary \& Waring 2015).

La dinámica de la regeneración establecida posterior al año semillero, a dos años y tres meses de la quema, y a cuatro años del incendio, muestra una disminución considerable de la densidad (Figura 6). Para el caso de la quema, la disminución fue muy abrupta entre la primera y segunda evaluación (septiembre 2011 y septiembre 2012), decreciendo en menor proporción para la tercera evaluación (marzo 2013); mientras que en la zona de incendio, la disminución en la regeneración fue importante entre las tres evaluaciones, atribuyendo en parte este fenómeno a las fuertes heladas que se presentan en la región, a pesar de la protección que crean el sotobosque y la hojarasca presentes en los distintos tratamientos, con el primero a mayor densidad en la quema prescrita. Al respecto, Madrigal et al. (2005) encontraron que la insolación, la competencia interespecífica y el área basal de los árboles semilleros fueron los factores determinantes para predecir la probabilidad de sobrevivencia del renuevo de $P$. pinaster, a tres años de su establecimiento; la densidad del renuevo disminuyó en 57.2, 68.6 y $77.6 \%$ en el primero, segundo y tercer años, respectivamente, con relación al renuevo inicial que fue de 10,533 plántulas $\mathrm{ha}^{-1}$. La reducción de la densidad en poblaciones forestales coetáneas conforme crece, obedece a varios factores limitativos, pero entre ellos destaca la reducción de espacio de crecimiento, que genera competencia intraespecífica y autoaclareo (Burkhart 2013).

Aunque los porcentajes de mortalidad del renuevo en el presente estudio son altos, las densidades en las dos zonas sometidas al fuego son todavía elevadas, por lo que se considera que aún habrá una disminución importante respecto al número inicial de plántulas establecidas después de la liberación de la semilla que le dio origen.
Como conclusiones, el fuego favoreció el establecimiento de la regeneración natural de $P$. montezumae en el área estudiada. Se demostró que la capa de hojarasca es un factor limitante en el establecimiento de la regeneración, evitando que la semilla se establezca al no encontrar condiciones adecuadas, o en su caso que la raíz llegue a suelo mineral, sufriendo así un estrés hídrico y consecuentemente la muerte. Sin embargo, ya establecidas las plántulas, la hojarasca las protege de las heladas.

Las quemas prescritas representan una alternativa viable para los silvicultores y gestores de ecosistemas que dependen del fuego, como es el caso del bosque de $P$. montezumae, logrando la renovación del bosque, considerando las altas densidades que se encuentran en la zona de quema, en comparación con la zona testigo.

Se halló que la densidad de regeneración tiende a disminuir a través del tiempo, debido posiblemente a diversos factores, como las bajas temperaturas invernales y la competencia intraespecífica, por lo que se estima que dicha disminución continuará. Asimismo, los niveles de radiación solar presentes favorecieron la regeneración post fuego.

Algunas recomendaciones se desprenden del presente estudio: debido a que el establecimiento de la regeneración se presentó posterior a un año semillero, se recomienda hacer uso de fuego prescrito a bajas intensidad y severidad, con anticipación a la dispersión de semilla. Se recomienda no realizar quemas prescritas de intensidad y severidad altas, para evitar otras consecuencias adversas potenciales, como la erosión del suelo y la presencia de plagas, entre otras.

\section{Literatura citada}

Arno SF. 2000. Fire in western forest ecosystems. In: Brown JK, Kapler-Smith J, eds. Wildland Fire in Ecosystems. Effects of Fire on Flora. USA: USDAForest Service-Rocky Mountain Research Station. pp. 97-120. https://www.fs.fed.us/rm/pubs/rmrs_gtr042_2.p df (accessed February 16, 2020).

Bermúdez-Rodríguez L. 2018. Efecto de la Extracción Forestal sobre la Estructura y Regeneración del Bosque de Pinus hartwegii en el Nevado de Toluca. MSc. Thesis, Universidad Autónoma del Estado de México.

Bonilla-Vichot M, Valdez L, Martínez-Becerra LW. 2009. Regeneración natural de Pinus tropicalis Morlet y vegetación asociada después de un incendio. Cerne 15: 215-220.

Bonnet VH, Schoettle AW, Shepperd WD. 2005. Postfire environmental conditions influence the spatial pattern of regeneration of Pinus ponderosa. Canadian Journal of 
Forest Research 35: 37-47. DOI: https://doi.org/10.1139/ $\underline{\mathrm{x} 04-157}$

Burkhart HE. 2013. Comparison of maximum size-density relationships based on alternate stand attributes for predicting tree numbers and stand growth.Forest Ecology and Management 289: 404-408. DOI: https://doi.org/ $\underline{\text { 10.1016/j.foreco.2012.10.041 }}$

DeBano LF, Neary DG, Folliott PF. 1998. Fire's Effects on Ecosystems. New York: Wiley. ISBN: 978-0-471-16356-5

De las Heras-Ibáñez J, Alfaro-Sánchez R, HernándezTecles EJ, Hedo J, Moya D. 2011. Restauración y manejo de pinares de pino carrasco tras incendio en el sureste de la Península Ibérica. Boletín del CIDEU 10: 63-79.

Egger K, Arocena J, Green S, Kennedy N, Massicotte H, Tackaberry L, Scholefield S. 2009. Assessment of Postbeetle Impacts on Natural Regeneration of Lodgepole Pine. Mountain pine beetle working paper 2009-07. Canada: Natural Resources Canada, Canadian Forest Service, Pacific Forestry Centre. ISBN: 9780100131450

Flores-Garnica JG, Benavides-Solorio JD. 2009. Efecto del fuego en la regeneración natural de ecosistemas forestales. In: Flores-Garnica JG, coord. Impacto Ambiental de Incendios Forestales. México: Mundi Prensa, pp. 141-152. ISBN: 978-607-7699-03-3.

González-Rosales A, Rodríguez-Trejo DA. 2004. Efecto del chamuscado de copa en el crecimiento en diámetro de Pinus hartwegii Lindl. en el Distrito Federal, México. Agrociencia 38: 537-544.

Haffey C, Sisk TD, Allen CD, Thode AE, Margolis EQ. 2018. Limits to ponderosa pine regeneration following large high-severity forest fires in the United States Southwest. Fire Ecology 14: 143-163. DOI: https:// doi.org/10.4996/fireecology.140114316

Hancock MH, Summers RW, Amphlett A, Willi J. 2009. Testing prescribed fire as a tool to promote scots pine Pinus sylvestris regeneration. European Journal of Forest Research 128: 319-333. DOI: https://doi.org/ 10.1007/s10342-009-0267-5

Hardesty J, Myers RL, Fulks W. 2005. Fire ecosystems and people: a preliminary assessment of fire as a global conservation issue. The George Wright Forum 22: 78-87. http://www.georgewright.org/224hardesty.pdf (accessed March 18, 2020).

INEGI [Instituto Nacional de Estádistica Geografía e Informática]. 1992. Síntesis Geográfica del Estado de Hidalgo. Aguascalientes, México: INEGI. ISBN: 968-892-711-2.

Jardel-Peláez E, Ramírez-Villeda R, Castillo-Navarro F, García-Ruvalcaba S, Balcázar OE, Chacón-Mathieu JC, Morfin-Ríos JE. 2006. Manejo del fuego y restauración de bosques en la Reserva de la Biosfera Sierra de
Manantlán, México. In: Flores-Garnica JG, RodríguezTrejo DA, eds. Incendios Forestales. México: Mundi Prensa, Comisión Nacional Forestal. pp. 214-242. ISBN: 968-7462-41-8.

Juárez-Martínez A, Rodríguez-Trejo DA. 2004. Efecto de los incendios forestales en la regeneración de Pinus oocarpa var. ochoterenae. Revista Chapingo. Serie Ciencias Forestales y el Ambiente 10: 125-130.

Li J, Li J. 2003. Regeneration and restoration of broadleaved Korean pine forests in Lesser Xing'an Mountains of Northeast China. Acta Ecologica Sinica 23: 1268-1277. DOI: https://doi.org/10.1007/s11676-012-02 94-9

López G, Pérez J, Kleinn C. 2001. SAS: Aplicaciones en el Campo Agropecuario y de los Recursos Naturales. Costa Rica: Centro Agronómico Tropical de Investigación y Enseñanza (CATIE). http://orton.catie.ac.cr/repdoc/A68 47e/A6847e.pdf (accessed February 22, 2020).

Madrigal J, Hernando C, Martínez E, Guijarro M, Díez C. 2005. Regeneración post-incendio de Pinus pinaster Ait. en la Sierra de Guadarrama (Sistema Central, España): modelos descriptivos de los factores influyentes en la densidad inicial y la supervivencia. Investigación Agrararia. Sistemas y Recursos Forestales 14: 36-51

Marroquín-Flores RA, Jiménez-Pérez J, Garza-Ocañas F, Aguirre-Calderón O, Estrada-Castillón E, Bourguet-Díaz R. 2007. Regeneración natural de Pinus pseudostrobus en zonas degradadas por incendio. Ciencia UANL 10: 33-37.

Martínez-Becerra LW. 2006. Uso de Quemas Prescritas en Bosques Naturales de Pinus tropicalis Morelet en Pinar del Río. PhD Thesis, Universidad de Pinar del Río.

Miller M. 2000. Fire autoecology. In: Brown JK, Smith JK, eds. Wildland Fire in Ecosystems. Effects of fire on flora. USA: USDA-Forest Service-Rocky Mountain Research Station. pp. 9-34. https://www.fs.fed.us/rm/pubs/rmrs gtr042 2.pdf (accessed February 23, 2020).

Mostacedo B, Fredericksen TS. 2000. Manual de Métodos Básicos de Muestreo y Análisis en Ecología Vegetal. Santa Cruz, Bolivia: Bolfor. http://www.bio-nica.info/ biblioteca/mostacedo2000ecologiavegetal.pdf (accessed February 14, 2010).

Ouzts J, Huffman D, Meador AS. 2015. Post-fire ponderosa pine regeneration with and without planting in Arizona and New Mexico. Forest Ecology and Management 354: 281-290. DOI: https://doi.org/10.1016/j.foreco.2015. 06.001

Pérez-Pereda CE, Durán-Manual F, Martínez-Becerra LW, Ramos-Rodríguez MP, Tamayo-Echevarría W. 2012. Regeneración natural de Pinus cubensis Griseb. en Guantánamo al aplicar quemas prescritas. Revista Forestal Baracoa 31: 15-22. 
Platt WJ. 1999. Southeastern pine savannas. In: Anderson RC, Fralish JS, Baskin JM, eds. Savannas, Barrens, and Rock Outcrop Plant Communities of North America. Cambridge: Cambridge University Press. pp. 23-51. ISBN: 978-0521035811.

Rodríguez-Trejo DA. 1996. Incendios Forestales. México: Mundi Prensa, UACH. ISBN: 968-7462-04-3.

Rodríguez-Trejo DA. 2008. Fire ecology, fire regimes and fire management in Mexico. Ambio 37: 548-556. DOI: https://doi.org/10.1579/0044-7447-37.7.548

Rodríguez-Trejo DA. 2014. Incendios de Vegetación: Su Ecología, Manejo e Historia. Vol. 1. México: Colegio de Posgraduados - Universidad Autónoma Chapingo, Academia Nacional de Ciencias Forestales, Asociación Mexicana de Profesionales Forestales, Parque Nacional Iztaccíhuatl-Popocatépetl, Comisión Nacional Forestal, Comisión Nacional de Áreas Naturales Protegidas. ISBN: 978-607-715-237-8.

Rodríguez-Trejo DA, Fulé PZ 2003. Fire ecology of Mexican pines and a fire management proposal. International Journal of Wildland Fire 12: 23-37. DOI: https://doi.org/10.1071/WF02040

Rodríguez-Trejo DA, Martínez-Muñoz P, Martínez Lara PJ. 2019. Efectos del fuego en el arbolado de un bosque tropical de pino y en el de una selva baja caducifolia en Villaflores, Chiapas. Ciencia Florestal 29: 1033-1047. DOI: https://doi.org/10.5902/1980509833952

Editor de sección: Enrique Jurado

Contribución de los autores: DDH concepción del estudio, muestreo en campo, apoyo en análisis estadístico, escritura; RRL concepción del estudio, muestreo en campo, escritura; DART análisis estadístico, escritura, apoyo en concepción y en muestreo en campo; AAS apoyos en campo, manejo de bases de datos y en escritura; CCMM apoyos en trabajo de campo, análisis estadístico $\mathrm{y}$ en escritura.
Ruano I, del Peso C, Bravo F. 2015. Post dispersal predation of Pinus pinaster Aiton seeds: key factors and effects on belowground seed bank. European Journal of Forest Research 134: 309-318. DOI: https://doi.org/ $\underline{10.1007 / \mathrm{s} 10342-014-0853-\mathrm{Z}}$

SAS. 2012. Statistical Analysis System. V.9.1 ed. SAS. Institute. Inc. N.C., USA: Cary.

Scott AC. 2018. Burning Planet. The Story of Fire Trough Time. Great Britain: Oxford University Press. ISBN: 9780198734840

Scott AC, Bowman DMJS, Bond WS, Pyne SJ, Alexander ME. 2014. Fire on Earth. Singapore: Wiley Blackwell. ISBN: 978-1-119-95357-9

Smith DM, Larson BC, Kelty MJ, Ashton PMS. 1997. The practice of silviculture. New York: Wiley . ISBN: 978-0471109419.

Vela-Correa G, Vázquez-Martínez BE, Rodríguez-Gaminio, ML, Domínguez-Rubio IV. 2007. Caracterización edáfica de sitios con regeneración natural de Pinus montezumae Lamb. en el volcán la Malinche, México. Agrociencia 41: 371-383.

Vela-Gálvez L. 1980. Contribución a la Ecología de Pinus patula. Publicación Especial 19, Instituto Nacional de Investigaciones Forestales. México.

Zachary T, Waring KM. 2015. Enhancing resiliency and restoring ecological attributes in second-growth Ponderosa pine stands in Northern New Mexico, USA. Forest Science 61: 93-12. DOI: https://doi.org/10.5849/ forsci.13-085. 